・研究报告・

\title{
垂叶榕榕小蜂群落及种间互作网络季节动态
}

\author{
董乙 $X^{1,2}$ 彭䎦琼 $^{1}$ 王 波 ${ }^{*}$ \\ 1 (中国科学院西双版纳热带植物园热带森林生态学重点实验室, 云南预腊 666303) \\ 2 (中国科学院大学, 北京 100049)
}

摘要: 群落中的物种相互作用构成了复杂的生态网络。有关物种的数量和组成的季节性动态变化已有较多的研究, 但是对于生态网络的动态变化知之甚少。揭示生态网络的动态变化对于理解群落的稳定性以及群落的动态变化过 程和机理具有重要意义。本研究以垂叶榕(Ficus benjamina)榕小蜂群落为研究对象, 分别在西双版纳的干季和雨季 采集了榕小蜂的种类和数量信息。比较了两个季节榕小蜂群落的动态变化以及共存网络的参数(例如网路直径、 连接数、嵌套性和群落矩阵温度)变化。结果显示: 雨季榕果内传粉榕小蜂Eupristina koningsbergeri所占比例高于 干季, 传粉榕小蜂的种群数量也高于干季, 而在干季非传粉榕小蜂的种类增加(干季15种小蜂, 雨季14种)。从榕树 一传粉榕小蜂互利共生系统的适合度来看, 干季非传粉小蜂的增加对传粉榕小蜂和榕树的适合度是不利的。在干 季, 共存网络物种间的连接数(干季 0.95 , 雨季 0.47 )多于雨季, 群落矩阵温度 (干季 23.24 , 雨季 2.64 ) 也显著高于雨 季。表明干季榕小蜂群落组成及种间关系较雨季更为复杂而多样, 高的矩阵温度暗示群落受到的干扰更大。

关键词：榕树-榕小蜂；昆虫群落; 生态网络; 季节动态; 种间互作

\section{Seasonal dynamics of fig wasp community and interaction networks in Ficus benjamina}

Yiyi Dong ${ }^{1,2}$, Yanqiong Peng ${ }^{1}$, Bo Wang ${ }^{1 *}$

1 Key Laboratory of Tropical Forest Ecology, Xishuangbanna Tropical Botanical Garden, Chinese Academy of Sciences, Mengla, Yunnan 666303

2 University of Chinese Academy of Sciences, Beijing 100049

\begin{abstract}
Species form complex interactive networks within communities, which often experience seasonal species oscillations or species composition shifts. However, the variation that occurs within such networks is poorly understood. Studying seasonal changes of ecological networks may reveal the mechanisms that maintain the stability of a community. In this study, we collected fig wasps from Ficus benjamina during the dry and rainy seasons in Xishuangbanna to understand how fig wasp communities may shift seasonally. We compared fig wasp abundance, species composition, and metrics of coexistence networks (e.g. network diameter, connectance, nestedness, and community temperature) between the rainy season and the dry season. The pollinating wasp, Eupristina koningsbergeri was the most abundant species in both rainy season and dry season. There were more non-pollinating fig wasp species during the dry season (15 fig wasp species) than the rainy season (14 fig wasp species). A higher number of non-pollinating fig wasps can be detrimental to fig-pollinator wasp mutualisms because they reduce both fig seed production and pollinator abundance. Additionally, the dry season experiences higher species connectivity ( 0.95 in dry season, 0.47 in rainy season), higher network temperature (23.24 in dry season, 2.64 in rainy season) than the rainy season, which indicate more complex inter-specific interactions, and a higher level of disturbance of fig wasp community in the dry season.
\end{abstract}

Key words: fig-fig wasp; insect community; ecological network; seasonal variation; species interaction

群落中共存的物种形成了互相作用的复杂网

络, 这样的互作网络反映了生态系统的作用过程,

收稿日期: 2019-09-19; 接受日期: 2020-01-13

基金项目: 国家自然科学基金(31570418; 31770463)、云南省基金(2016FB050)和中国科学院 “一三五”规划(2017XTBG-T01)

* 通讯作者 Author for correspondence. E-mail: wangbo@xtbg.ac.cn 
是生态系统产出和服务的基础(Hagen et al, 2012)。 互利共生网络 (mutualistic networks)和对抗网络 (antagonistic networks)是两种主要的生态网络类型 (Olesen, et al, 2012)。对抗网络包括传统的食物网和 宿主-寄生者网络(Henri \& Veen, 2011; Jacob et al, 2011); 互利共生网络包括植物-访花者(传粉者)网 络(Memmott, 1999)、植物种子散布者网络(Donatti et al, 2011; Schleuning et al, 2011)和寄主-共生者网络 (Guimarães et al, 2007)。这些类型代表了目前生态 网络研究的主要对象。

网络的基本组成是节点(node)和连接(link)。在 生态网络中, 节点可能是生物个体、物种、种群、 种团、功能团或整个群落(Hagen et al, 2012)。网络 结构的常见测量指标包括物种和连接数以及连接 的分布(见Box 1), 其中一些测量指标与网络的复杂 度、稳定性和资源分类具有某种联系(Williams \& Martinez, 2000; Berlow et al, 2009)。网络同时表现出 一些可辨别的亚结构模式, 通常表现为分形状模式 (fractal-like manner) (Olesen et al, 2007; Stouffer \& Bascompte, 2010)。例如, 食物网可以被分解成食物 链, 三级营养关系最终分解成成对的种间互作关系
(Woodward et al, 2012)。嵌套性和模块性是两个得 到了较多关注的网络结构测度(Lewinsohn et al, 2006; Olesen et al, 2007)。在一个嵌套的网络中, 特 化物种间的连接是泛化物种间连接的子集(Bascompte et al, 2003)。模块性描述了物种的子集，其 内部具有高的连接数, 但是与其他子集之间连接较 少(Olesen et al, 2007)。嵌套性和模块性常常被认为 是互相排斥的(Lewinsohn et al, 2005), 但是也存在 反例(Olesen et al, 2007; Fortuna et al, 2010)。

物种的丰富度、物种组成以及多样性随时间的 变化是一个自然过程，决定着种间互作网络的基本 特征(节点、连接数量、嵌套性和模块性等) (Krishna, et al, 2008)。伴随着群落中物种种群大小的季节性 改变，一些物种之间的连接断裂，而与另一些物种 形成新连接。任何明显的时空干扰都可能传递到物 种互作网络，导致更进一步的物种种群大小和种间 连接的改变(Santos et al, 2014)。利用网络对群落进 行分析, 能为理解一个群落中物种间互作关系的动态 变化和解释生态系统对干扰的响应提供新的视角。

榕树是热带雨林中的关键类群。每种榕树都有 专性的传粉榕小蜂为其传粉，同时也为多种非传粉

\section{Box 1 网络分析术语}

节点(node): 网络中的节点, 可以是物种、种群、种团、功能团等。

边(edge)：连接两个节点的连线，暗示节点间存在联系。

网络大小(size): 网络中节点的数量。

连接数/度(connectance): 是网络中物种之间实际连接数之和占总的可能连接数的比例, 取值范围在 $0-1$, 数值越大表

明网络中节点之间的连接性越大。

连接的分布: 节点连接数的分布，常表现出幂律分布。

直径(diameter)：计算网络中任意两个节点之间的最短距离，其中的最大值称为网络的直径。

平均路径长度(average path length)：网络中任意两个节点之间的距离的平均值。

加权嵌套性(weighted nestedness metric based on overlap and decreasing fill, WNODF): 考虑了互作频率的嵌套性指标。取 值为 $0-1,0$ 表示完全混乱, 1表示完全嵌套。

模块性(modularity): 是关于网络节点分区(或节点类型)的指标, 测量了分区(节点类型)的好坏程度或者不同的节点类 型彼此之间的分离程度。

全局聚类系数(global clustering coefficient): 是反映网络中节点紧密关系的参数, 也称为传递性，表征了整个网络的平 均“成簇性质”。节点 $\mathrm{i}$ 的聚类系数为节点 $\mathrm{i}$ 的邻接点之间实际存在的边数与所有可能的边数的比值。网络的全局聚类系数为 所有节点的聚类系数的均值。

群落矩阵温度(temperature, T)：实际观测的群落分布结构与完全嵌套结构的群落分布的偏离程度，用于表征群落的无 序性。 $T$ 值的大小表示实际观测的群落分布结构与完全嵌套结构的群落分布的偏离程度: $T=0$, 则是完全嵌套; $T=100$, 则 是完全随机无序。一个群落的温度越低, 表示群落越稳定。较高的温度代表群落受到的千扰较大。 
榕小蜂提供了生长、发育的场所。这些非传粉榕小 蜂是包括造掼者(能将卵产在子房中致使子房膨大 变为瘘花)、寄居者(将卵产于其他造瘃者产卵的瘞 花子房内)和复寄生者(能将卵产于其他小蜂体内) 在内的多种榕小蜂，它们属于不同的营养级(Herre, 1999; Kerdelhué et al, 2000)。因此, 一个榕小蜂群落 是包括了多种榕小蜂以及多营养级的复杂而相对 独立的群落, 是研究昆虫种间互作网络的理想模 型。气候变化可能会打破榕树-榕小蜂的互利共生 关系, 甚至导致传粉榕小蜂灭绝, 进而影响到其他 营养级水平的物种组成(Jevanandam et al, 2013)。

本研究调查了西双版纳预仑地区垂叶榕(Ficus benjamina)上榕小蜂群落的组成, 比较了垂叶榕榕 小蜂群落物种丰富度及共存网络的季节动态变化, 讨论了群落的物种丰富度和共存网络结构之间的 关联。通过对上述数据的分析试图回答: (1)榕小蜂 群落物种组成和丰富度存在怎样的季节动态变化?

(2)㭲小峰共存网络(歕套性和连接数) 是否存在明显 的季节变化? (3)物种的丰富度和网络结构之间具有 怎样的关联?

\section{材料与方法}

\section{1 样点概况}

研究样地位于云南省西双版纳傣族自治州(约 $21^{\circ} 08^{\prime}-22^{\circ} 35^{\prime} \mathrm{N}, 9^{\circ} 56^{\prime}-101^{\circ} 50^{\prime} \mathrm{E}$ )。该地区属于热 带季风型气候, 全年的气温相对稳定, 年平均气温 为 $21.8^{\circ} \mathrm{C}$, 一年中降水量变化显著, 根据降水量、温 度和湿度的变化可把一年分为雨季(6-10月)和干季 (11月至翌年5月) (张克映, 1963)。来自云南西双版 纳森林生态系统国家野外科学观测研究站 (http://bnf.cern.ac.cn/)的数据显示：2002-2018年间, 雨季的降水量为 $7.19 \pm 2.81 \mathrm{~mm}$, 温度为 $25.37 \pm$ $1.08^{\circ} \mathrm{C}$, 湿度为 $82.08 \pm 4.37 \%$, 干季的降水量为 3.97 $\pm 5.10 \mathrm{~mm}$, 温度为 $20.69 \pm 3.14^{\circ} \mathrm{C}$, 湿度为75.73 \pm $8.46 \%$ (均值土标准差)。

\section{2 垂叶榕及其榕小蜂群落}

垂叶榕隶属于桑科榕属, 雌雄同株。榕果对生 或单生叶腋, 成熟时呈黄色或红色。综合文献报道, 垂叶榕上的榕小蜂隶属于膜翅目小蜂总科中 4 科 8 属 18 种, 单批次果出蜂种类最多为 14 种。群落中 小蜂种类信息及营养级水平见附录 1 (白莉芬等, 2006; 王振吉, 2010)。

\section{3 样品采集}

2018年, 我们在雨季和干季收集了西双版纳傣 族自治州预仑地区的垂叶榕榕小蜂。每个季节在至 少3株榕树上收集榕果，每棵树上采集30个雄花期 的榕果(果壁变软、颜色发黄)带回实验室。将单个 榕果沿纵轴剖开，置于 120 目的纱网袋中。待小蜂羽 化进入袋内后, 收集所有小蜂, 保存于盛有无水乙 醇的样品瓶中。2002年垂叶榕榕小蜂群落数据来自 白莉芬等(2006)的采样，采样季节、方法及小蜂分 类、计数流程与本实验相同。

\section{4 榕小蜂的群落调查}

使用体视显微镜(江南JSZSB-HG785940)对每 个榕果内的榕小蜂进行分类、鉴定并统计数量(白莉 芬等, 2006; 王仲敏等, 2010)。

\section{5 数据分析}

\subsection{1 不同季节垂叶榕榕小蜂群落物种组成及丰 富度比较}

整合了白莉芬等于2002年对垂叶榕榕小蜂群 落的调查结果, 其中 90 个榕果数据来自本次采样, 30 个榕果数据来自白莉芬等(2006)。计算垂叶榕榕 小蜂在干季和雨季各物种的比例。采用相似性分析 (analysis of similarity, ANOSIM)检验垂叶榕榕小蜂 群落物种在不同季节物种的组成是否具有显著差 异。ANOSIM分析使用了非参数的置换检验(permutation), 其过程是基于一个相似性矩阵检验组间 差异的显著性比较。此处, 群落间相似性矩阵采用 Bray-Curtis 距离进行了 999 次置换检验 (Clarke, 1993)。

\subsection{2 不同季节榕小蜂共存网络的比较}

榕小蜂共存网络分析可以图形化显示榕小蜂 的种间共存关系。首先将榕小蜂群落数据转换成 $0 / 1$ 矩阵(“0”表示物种不存在, “ 1 ”表示物种存在), 进一 步将 $0 / 1$ 矩阵转换成二分网络, 最后通过 $R$ 语言 igraph包获得榕小蜂物种间的邻接矩阵并绘制网络 图。网络中每个节点的颜色表示营养级水平，每条 边(edge)的权重(weight)代表了物种间的连接频率。 同时比较了两个季节的网络大小(size)、全局聚类 系数、连接性(Wasserman \& Faust, 1995)、矩阵温 度(T) (Atmar \& Patterson, 1993; Almeida-Neto et al, 2007) 以及加权嵌套性 (weight nestedness metric based on overlap and decreasing fill, WNODF)。

采用R 3.5.2 (R Development Core Team, 2018) 
表1 西双版纳地区垂叶榕榕小蜂物种丰富度(均值 \pm 标准差, $N=120$ )。其中90个榕果数据来自本次采样, 30 个榕果数据来自 白莉芬等(2006)。

Table 1 Species abundance and composition of fig wasps in Ficus benjamina in Xishuangbanna (mean \pm SD, $N=120$ ). Of the 120 figs, 90 were from our study, the other 30 were from Bai (2006).

\begin{tabular}{|c|c|c|c|c|c|}
\hline \multirow[t]{2}{*}{ 榕小蜂 Fig wasp } & \multirow{2}{*}{$\begin{array}{l}\text { 可能的营养级水平 } \\
\text { Presumble trophic level }\end{array}$} & \multicolumn{2}{|l|}{ 干季 Dry season } & \multicolumn{2}{|l|}{ 雨季 Rainy season } \\
\hline & & 丰富度 Richness & 百分比 \% & 丰富度 Richness & 百分比 \% \\
\hline Eupristina koningsbergeri & 传粉者 Pollinator & $129.01 \pm 83.22$ & 72.24 & $308.39 \pm 183.62$ & 93.18 \\
\hline Acophila sp. 1 & 造㾉者 Galler & $0.32 \pm 1.17$ & 0.18 & $0.09 \pm 0.44$ & 0.03 \\
\hline Ormyrus sp. 1 & 寄生者 Parasitoid & $0.46 \pm 1.54$ & 0.26 & $0.01 \pm 0.09$ & $<0.01$ \\
\hline Philotrypesis sp. 1 & 寄生者 Parasitoid & $6.14 \pm 10.40$ & 3.44 & $17.95 \pm 25.62$ & 5.42 \\
\hline Philotrypesis sp. 2 & 寄生者 Parasitoid & $1.37 \pm 3.04$ & 0.77 & $1.12 \pm 4.54$ & 0.34 \\
\hline Philotrypesis sp. 3 & 寄生者 Parasitoid & $1.29 \pm 4.69$ & 0.72 & - & - \\
\hline Philotrypesis tridentate & 寄生者 Parasitoid & $7.11 \pm 8.96$ & 3.98 & $0.14 \pm 1.06$ & 0.04 \\
\hline Sycobia sp. 1 & 造搖者 Galler & $2.67 \pm 4.59$ & 1.50 & $0.14 \pm 0.63$ & 0.04 \\
\hline Sycobia sp. 2 & 造㾉者 Galler & $1.43 \pm 3.73$ & 0.80 & $0.01 \pm 0.09$ & $<0.01$ \\
\hline Sycobia sp. 3 & 造㾉者 Galler & $1.51 \pm 2.71$ & 0.85 & $0.25 \pm 1.39$ & 0.08 \\
\hline Sycophila sp. 1 & 寄生者 Parasitoid & $1.57 \pm 3.52$ & 0.88 & $0.27 \pm 1.05$ & 0.08 \\
\hline Sycoscapter sp. 1 & 寄生者 Parasitoid & $12.62 \pm 26.64$ & 7.06 & $0.46 \pm 2.10$ & 0.14 \\
\hline Walkerella benjamini & 造㾉者 Galler & $12.14 \pm 10.21$ & 6.80 & $1.90 \pm 5.43$ & 0.57 \\
\hline Walkerella sp. 1 & 造㾉者 Galler & $0.78 \pm 1.42$ & 0.43 & $0.19 \pm 1.10$ & 0.06 \\
\hline Walkerella sp. 2 & 造㾉者 Galler & $0.19 \pm 0.77$ & 0.11 & $0.04 \pm 0.30$ & 0.01 \\
\hline
\end{tabular}

的软件包包括vegan、 bipartite (Dormann et al, 2009)、 agricolae、dplyr、ggplot2、qgraph、igraph、reshape2 和psysh等, 以进行数据分析。

\section{2 结果}

\section{1 不同季节垂叶榕榕小蜂群落物种组成及丰富} 度比较

干季共收集到15种小蜂，雨季收集到14种(见 表1)。在雨季没有收集到Philotrypesis sp. 3这种寄生 蜂。ANOSIM检验结果显示干季和雨季垂叶榕榕小 蜂的群落组成发生了显著变化 $(R=0.28, P<0.01)$, 干季和雨季传粉榕小蜂占比分别为 $72.24 \%$ 和 $93.18 \%$ ，非传粉榕小蜂比例分别为 $27.76 \%$ 和 $6.82 \%$ 。 非传粉造瘘蜂Walkerella benjamini在干季和雨季的 比例分别为 $6.80 \%$ 和 $0.57 \%$ 。寄生蜂Philotrypesis sp. 1在干季和雨季的占比变化幅度不大, 分别为 $3.44 \%$ 和 $5.42 \%$; 而寄生蜂P. tridentate在干季(3.98\%)的所 占比例高于雨季 $(0.04 \%)$; 复寄生蜂Sycoscapter sp. 1在干季的占比(7.06\%)也高于雨季 $(0.14 \%)$ 。

垂叶榕传粉榕小蜂E. koningsbergeri在干季平 均单果出蜂达到 129.01 只 $(N=120)$, 在雨季平均 308.39 只, 两个季节的数量差异显著 $(\mathrm{df}=120, P<$
0.001)。Philotrypesis sp. 1 的数量也是雨季显著多于 干季 $(\mathrm{df}=120, P<0.001)$ 。而Sycoscapter sp. $1(\mathrm{df}=$ $120, P<0.001)$ 、Walkerella benjamini $(\mathrm{df}=120, P<$ $0.001)$ 和 $P$. tridentate $(\mathrm{df}=120, P<0.001)$ 的数量, 在干季显著多于雨季(图1)。

\section{2 不同季节榕小蜂共存网络的比较}

垂叶榕隐头果内所有榕小蜂物种进行共发生 网络分析结果表明(图2), 干季榕小蜂群落包含 15 个 节点, 100 条边, 连接数为 0.95 , 平均路径长度为 1.05 , 全局聚类系数为 0.96 。雨季榕小蜂群落包含 14 个节点, 43 条边, 连接数为 0.47 , 平均路径长度为 1.53 , 全局聚类系数为 0.61 。雨季的平均路径长度大 于干季 $(1.53>1.05)$ 。在两个季节网络中, 干季榕小 蜂群落各种榕小蜂(每个节点)的连接数为9-14, 而 雨季各种榕小蜂(每个节点)的连接数为 $2-13$ 。其中 传粉榕小蜂的连接数均为最多(干季: 14 , 雨季: 13)。

小蜂群落的矩阵温度在干季(23.24)高于雨季 (2.64)。干季和雨季的榕小蜂群落的加权嵌套性 WNODF数值分别为 33.57 和 31.78 。表明干季榕小蜂 群落与雨季相比更不稳定, 但干季榕小蜂群落的嵌 套性高于雨季。不同季节榕小蜂群落的各嵌套性和 网络指标的比较结果(图3)表明, 干季的群落矩阵温 


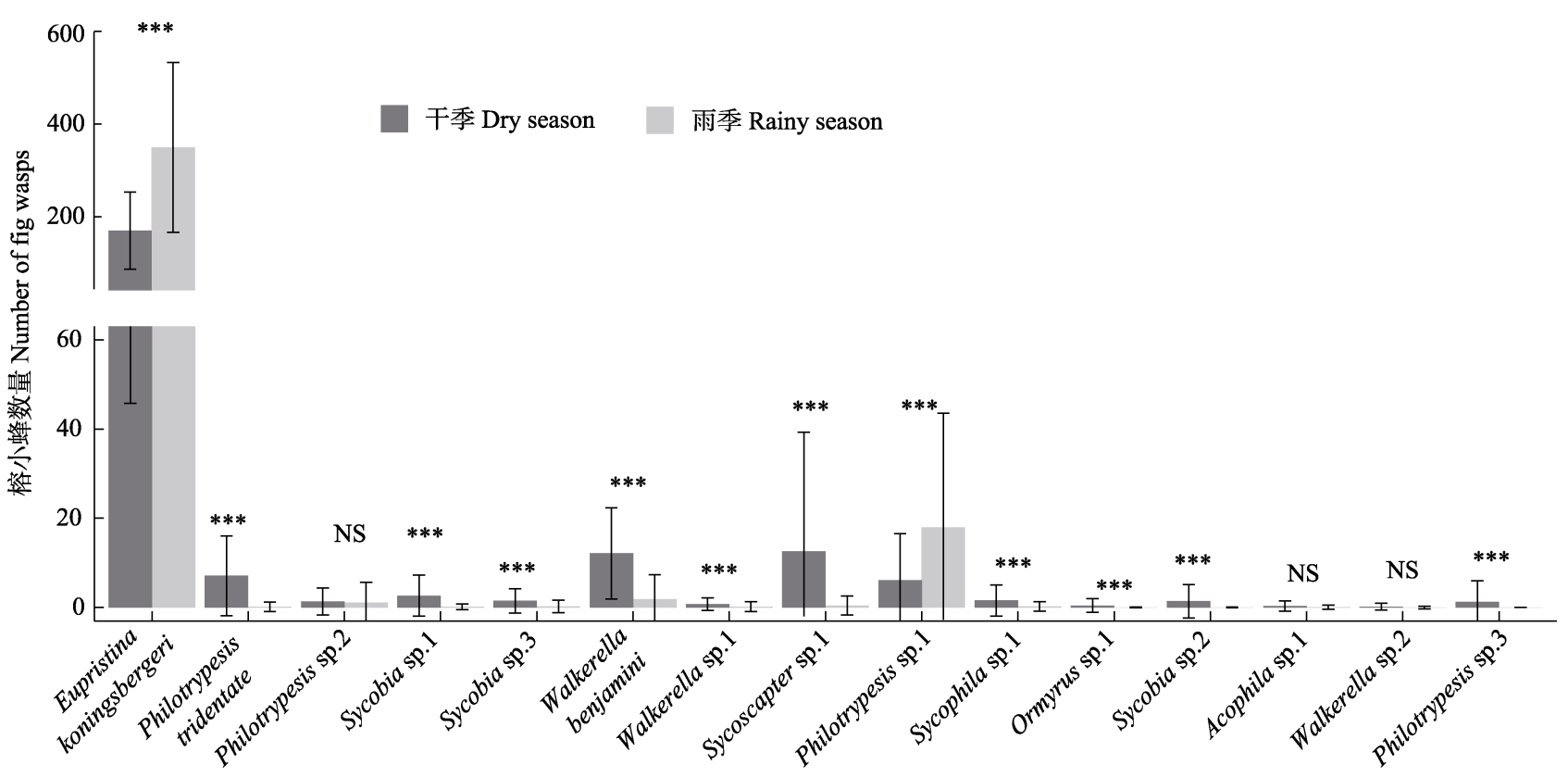

图1 西双版纳地区雨季和干季垂叶榕榕小蜂物种数量比较。采用独立样本 $t$-检验, 柱形图和误差线分别代表均值和标准差。 *** $\boldsymbol{P}<0.001$; NS表示不显著。

Fig. 1 Histogram of the fig wasp abundance of Ficus benjamina in Xishuangbanna. Two sample t-test was used to compared fig wasp abundance between rainy and dry seasons. Bars and errors represent mean \pm SD. $* * * P<0.001$; NS, Not significant.
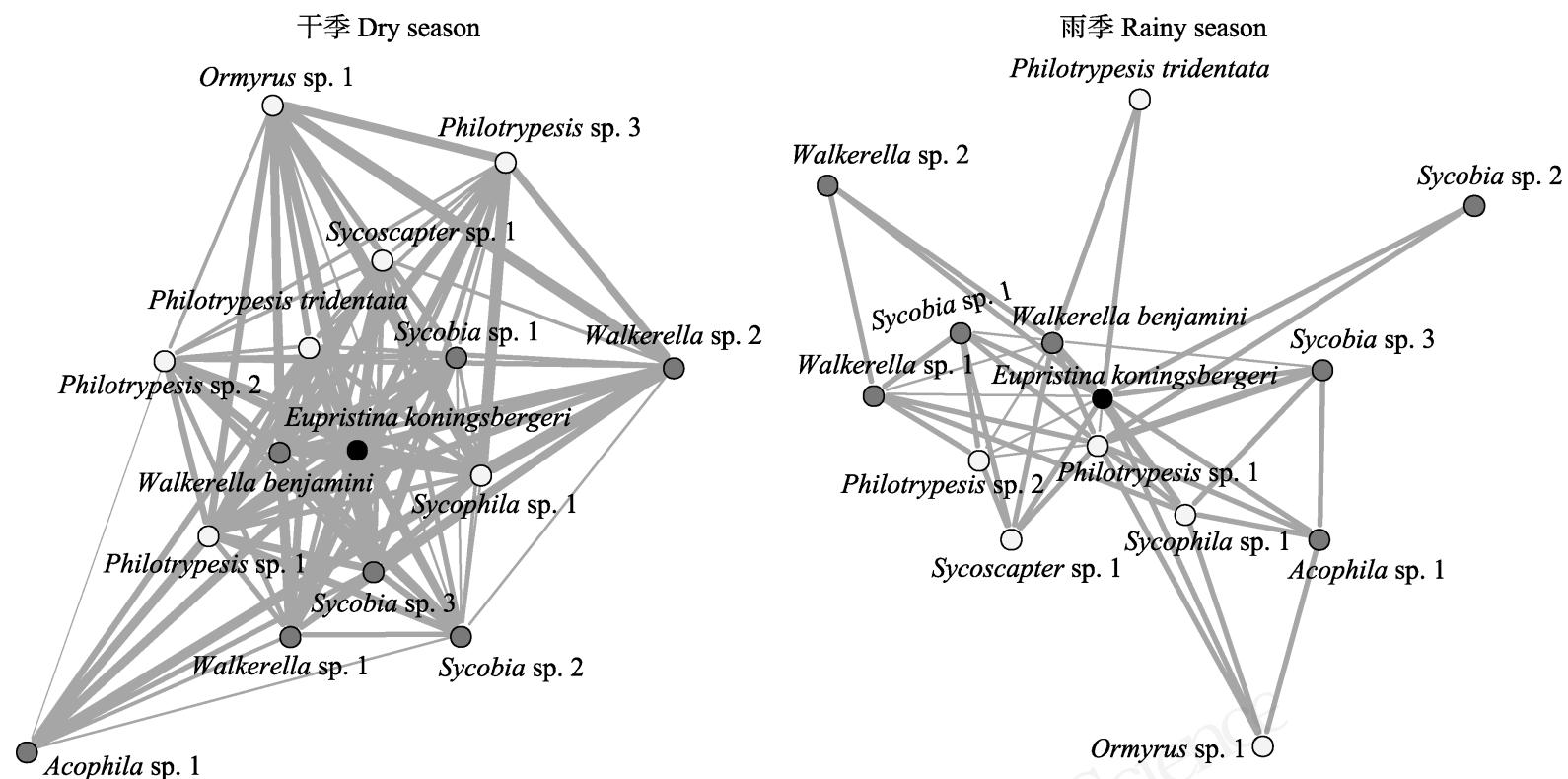

○传粉者 Pollinator ○造瘦者 Galler ○寄生者 Parasitoid

图2 西双版纳地区两个季节垂叶榕榕小蜂群落的加权共存网络。节点颜色代表可能的营养级水平, 边的宽度代表物种同时 出现的频率。

Fig. 2 The weighted co-occurrence networks of the wasp community of Ficus benjamina in Xishuangbanna. The nodes are colored by presumable trophic level, the width of each edge is proportional to the co-occur frequency of species.

度 $(\mathrm{df}=3, P<0.05)$ 、网络距离 $(\mathrm{df}=3, P<0.05)$ 和网 络的连接数 $(\mathrm{df}=3, P<0.05)$ 显著大于雨季; 而群落
的嵌套性 $(\mathrm{df}=3, P=0.31)$ 和网络直径 $(\mathrm{df}=3, P=$ 0.07)在两个季节没有显著差异。 


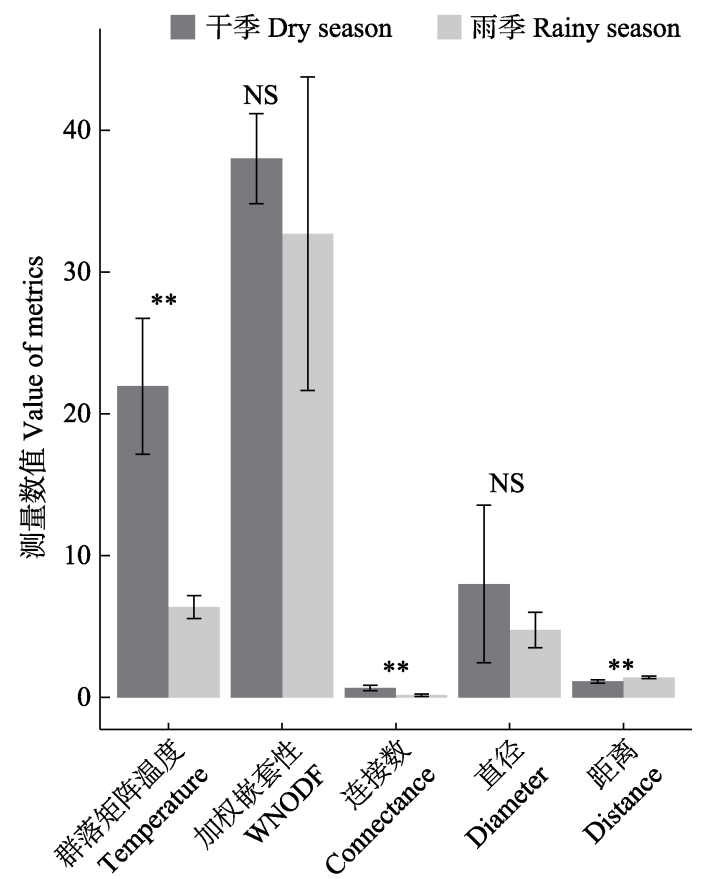

测量指标 Measurement of metrics

图3 西双版纳地区不同季节榕小蜂群落物种共存网络指标 的比较。使用 $t$ 检验比较了各指标两个季节的差异, 柱形图和 误差线代表平均值 \pm 标准差。** $P<0.01$; $* P<0.05$; NS表 示不显著。

Fig. 3 Fig wasp community co-occurrence network metrics between the dry and the rainy seasons in Xishuangbanna. The difference between the dry season and the rainy season were compared with $t$-test, the bars and error lines are mean $\pm \mathrm{SD}$. WNODF, Weight nestedness metric based on overlap and decreasing fill. ${ }^{* *} P<0.01$; $* P<0.05$; NS, Not significant.

\section{3 讨论}

本研究比较了不同季节垂叶榕隐头果内榕小 蜂共存网络的参数和群落的季节动态变化。结果发 现, 雨季榕果内传粉榕小蜂的占比和绝对数量均高 于干季，但干季非传粉榕小蜂的种类却多于雨季。 榕小蜂共存网络的分析进一步表明干季群落矩阵 温度和物种间的连接数显著高于雨季。以上结果均 暗示, 干季榕小蜂群落受到的干扰更大, 但小蜂群 落的组成及种间关系却较雨季更为复杂和多样。从 榕树一传粉榕小蜂互利共生系统的适合度角度来看, 干季非传粉小蜂比例和数量的增加对传粉榕小蜂 和榕树的互惠共生关系是不利的。

垂叶榕榕小蜂群落中包含了造瑤蜂、寄居蜂和 复寄生蜂。传粉榕小蜂是特殊的造㾉蜂, 在发育过 程中会取食胚珠, 并为榕树传粉, 所以传粉榕小蜂 与榕树之间存在着对雌花资源利用的权衡(Wang et al, 2013)。非传粉造㾉蜂(例如垂叶榕中Walkerella属 的造瘘蜂)从榕果外部产卵于雌花中, 不会为榕树 传粉。它们会与传粉榕小蜂竞争雌花资源, 它们的 产卵使得传粉榕小蜂需要花费更多的时间去搜寻 可产卵的雌花子房，降低了产卵率(白莉芬等, 2006; 王仲敏等, 2010; Cardona \& Kattan, 2019)。Sycoscapter和Philotrypesis属的非传粉小蜂是寄居类或复 寄生类小蜂。它们能将卵产于其他榕小蜂的瘞花中 或体内，与寄主竞争营养并导致寄主死亡(白莉芬 等, 2006)。寄生蜂一般以造曒蜂为寄主, 在一定数 量范围内, 寄生蜂的数量和寄主的数量呈正相关。 但是大量寄生蜂的存在会减少寄主的数量, 导致两 者数量呈负相关关系, 最终使得两者数量呈现复杂 的动态变化(Shi et al, 2011)。此外, 复寄生蜂Sycophila sp. 1、Ormyrus sp. 1和Sycoscapter sp. 1 能将卵产 于其他造瘞者产生的痚花子房内，且发育过程中以 榕果的胚珠为食, 导致榕树种子数量减少。因此, 非传粉造瘃蜂、寄生蜂和复寄生蜂的存在对传粉榕 小蜂和榕树的繁殖均会造成负面影响(Kerdelhué \& Rasplus, 1996; Weiblen, 2002; Cook \& Rasplus, 2003; Kong et al, 2016)。

垂叶榕榕小蜂的群落在干季和雨季具有显著 不同的物种数量和组成。传粉榕小蜂的比例在雨季 显著多于干季, 并且两个季节中传粉榕小蜂均为优 势种, 这与之前的研究报道结果一致(白莉芬等, 2006; 王仲敏等, 2010)。这暗示在垂叶榕榕小蜂群 落中, 传粉榕小蜂可能在资源的利用上占主导地位, 非传粉榕小蜂也需要依赖传粉榕小蜂维持其在榕 果中的生存。雨季的气候条件(温度高且降水量多) 可能不适于非传粉榕小蜂的寄主搜寻、产卵以及子 代的发育, 从而导致非传粉小蜂的比例较干季更少, 仅占 $6.82 \%$ 。在雨季, 榕果的发育速度比干季更快, 这有利于进入榕果内产卵的传粉榕小蜂。绝大多数 非传粉小蜂在果外产卵, 其产卵过程更容易受温度 和降水量等气候因素的干扰。而且雨季榕果发育速 率较快, 留给非传粉小蜂产卵的机会变少, 导致其 子代数量变少。有些非传粉榕小蜂属于稀有种, 发 生没有连续性, 且只在特定的月份或地区出现(王 仲敏等, 2010)。此外, 采样点周围的生境和采样数 量也会影响榕小蜂的种类和数量。基于两个季节垂 叶榕榕小蜂群落的物种组成以及物种数量的季节 变化, 我们推测小蜂群落可能存在分布区域上的动 
态变化, 暗示在更广泛的地域范围内, 可能存在榕 小蜂的集合群落(meta-community) (May et al, 2007)。

从网络的角度来看, 干季的垂叶榕榕小蜂群落 的物种组成共存网络拥有更高的群落温度和连接 数。高的群落温度暗示群落处于较强的干扰之下, 群落中数量稀少的物种消失的可能性极大。群落加 权嵌套性在两个季节没有显著差异, 但是相比于随 机群落, 两个季节的小蜂群落均呈现出极高的嵌套 性。干季非传粉小蜂的占比高于雨季, 进一步表明 干季群落中非传粉榕小蜂在榕果内的分布更为泛 化。此外, 干季的连接数、节点和网络直径也多于 雨季, 这表明干季榕小蜂物种间存在更多的关联、 群落也更复杂(Jacquet et al, 2016)。温度、降雨等气 候变化会影响榕小蜂对寄主的搜寻、榕果的发育历 程和榕小蜂群落组成(Zhang et al, 2006; Jevanandam et al, 2013), 进而影响网络参数特征。因此, 气候因 子的变化可能导致了榕小蜂种群数量、种间共存网 络的季节性动态变化。

榕小蜂群落的季节性动态变化与非生物因子 (温度和降水量等)和榕小蜂群落中物种间的相互作 用均有关。但仅从网络的测量指标(例如连接数、群 落温度和嵌套性等)来探究榕小蜂群落中网络的复 杂度和稳定性, 并不能完全解释群落或网络复杂度 的成因。因此, 结合种间互作和非生物因子对网络 复杂度进行分析, 有助于我们更好地理解群落或网 络结构。对网络复杂度和稳定性的进一步认识和分 析需要网络分析理论和方法上的创新。

\section{致谢：感谢张进媛在数据收集过程中的协助。}

\section{参考文献}

Almeida-Neto M, Guimarães PR Jr, Lewinsohn TM (2007) On nestedness analyses: Rethinking matrix temperature and anti-nestedness. Oikos, 116, 716-722.

Atmar W, Patterson BD (1993) The measure of order and disorder in the distribution of species in fragmented habitat. Oecologia, 96, 373-382.

Bai LF, Yang DR, Shi ZH, Peng YQ, Zhai SW (2006) Community structure of fig wasp in Ficus benjamina in different habitats. Biodiversity Science, 14, 340-344. (in Chinese with English abstract) [白莉芬, 杨大荣, 石章红, 彭艳琼, 翟树伟 (2006) 垂叶榕隐头果内小蜂群落结构与生境关 系的初步研究. 生物多样性, 14, 340-344.]

Bascompte J, Jordano P, Melián CJ, Olesen JM (2003) The nested assembly of plant-animal mutualistic networks. Proceedings of the National Academy of Sciences, USA, 100, 9383-9387.

Berlow EL, Dunne JA, Martinez ND, Stark PB, Williams RJ, Brose U (2009) Simple prediction of interaction strengths in complex food webs. Proceedings of the National Academy of Sciences, USA, 106, 187-191.

Cardona W, Kattan GH (2019) Complex effects of nonpollinating wasps on the relationship between pollinating wasp and seed production in Ficus andicola. Acta Oecologica, 98, 45-49.

Clarke KR (1993) Non-parametric multivariate analyses of changes in community structure. Australian Journal of Ecology, 18, 117-143.

Cook JM, Rasplus JY (2003) Mutualists with attitude: Coevolving fig wasps and figs. Trends in Ecology \& Evolution, 18, 241-248.

Donatti CI, Guimarães PR, Galetti M, Pizo MA, Marquitti FM, Dirzo R (2011) Analysis of a hyper-diverse seed dispersal network: Modularity and underlying mechanisms. Ecology Letters, 14, 773-781.

Dormann CF, Fründ J, Blüthgen N, Gruber B (2009) Indices, graphs and null models: Analyzing bipartite ecological networks. The Open Ecology Journal, 2, 7-24.

Fortuna MA, Stouffer DB, Olesen JM, Jordano P, Mouillot D, Krasnov BR, Poulin R, Bascompte J (2010) Nestedness versus modularity in ecological networks: Two sides of the same coin? Journal of Animal Ecology, 79, 811-817.

Guimarães PR Jr, Rico-Gray V, Oliveira PS, Izzo TJ, dos Reis SF, Thompson JN (2007) Interaction intimacy affects structure and coevolutionary dynamics in mutualistic networks. Current Biology, 17, 1797-1803.

Hagen M, Kissling WD, Rasmussen C, De Aguiar MA, Brown LE, Carstensen DW, Alves-Dos-Santos I, Dupont YL, Edwards FK (2012) Biodiversity, species interactions and ecological networks in a fragmented world. Advances in Ecological Research, 46, 89-210.

Henri DC, Van Veen FJF (2011) Body size, life history and the structure of host-parasitoid networks. Advances in Ecological Research, 45, 135-180.

Herre EA (1999) Laws governing species interactions? Encouragement and caution from figs and their associates. In: Levels of Selection in Evolution (ed. Keller L), pp. 209-237. Princeton University Press, Princeton.

Jacob U, Thierry A, Brose U, Arntz WE, Berg S, Brey T, Fetzer I, Jonsson T, Mintenbeck K, Möllmann C, Petchey OL, Riede JO, Dunne JA (2011) The role of body size in complex food webs: A cold case. Advances in Ecological Research, 45, 181-223.

Jacquet C, Moritz C, Morissette L, Legagneux P, Massol F, Archambault P, Gravel D (2016) No complexity-stability relationship in empirical ecosystems. Nature Communications, 7, 12573. 
Jevanandam N, Goh AG, Corlett RT (2013) Climate warming and the potential extinction of fig wasps, the obligate pollinators of figs. Biology Letters, 9, 20130041.

Kerdelhué C, Rasplus JY (1996) The evolution of dioecy among Ficus (Moraceae): An alternative hypothesis involving non-pollinating fig wasp pressure on the fig-pollinator mutualism. Oikos, 77, 163-166.

Kerdelhué C, Rossi JP, Rasplus JY (2000) Comparative community ecology studies on old world figs and fig wasps. Ecology, 81, 2832-2849.

Kong Y, Wang R, Yang DR, Sreekar R, Peng YQ, Compton SG (2016) Non-pollinator fig wasp impact on the reproductive success of an invasive fig tree: Why so little. Biocontrolence \& Technology, 26, 1432-1443.

Krishna A, Guimarães RP Jr, Jordano P, Bascompte J (2008) A neutral-niche theory of nestedness in mutualistic networks. Oikos, 117, 1609-1618.

Lewinsohn TM, Novotny V, Basset Y (2005) Insects on plants: Diversity of herbivore assemblages revisited. Annual Review of Ecology. Evolution, and Systematics, 36, 597-620.

Lewinsohn TM, Paulodo PI, Jordano P, Bascompte J, Olesen JM (2006) Structure in plant-animal interaction assemblages. Oikos, 113, 174-184.

May R, Mclean A (translated by Tao Y, Wang BH) (2007) Theoretical Ecology: Principles and Applications. Oxford University Press on Demand. [May R, Mclean A (陶毅, 王 百华译) (2010) 理论生态学一一原理及应用. 高等教育 出版社, 北京.]

Memmott J (1999) The structure of a plant-pollinator food web. Ecology Letters, 2, 276-280.

Olesen JM, Bascompte J, Dupont YL, Jordano P (2007) The modularity of pollination networks. Proceedings of the National Academy of Sciences, USA, 104, 19891-19896.

Olesen JM, Dupont YL, Hagen M, Rasmussen C, Trøjelsgaard K (2012) Structure and dynamics of pollination networks: The past, present, and future. In: Evolution of Plant-Pollinator Relationships (ed. Patiny S), pp. 374-391. Cambridge University Press, Cambridge.

R Development Core Team (2018) R: A Language and Environment for Statistical Computing. R Foundation for Statistical Computing, Vienna, Austria.

Santos GMM, Wesley D, Presley SJ (2014) The seasonal dynamic of ant-flower networks in a semi-arid tropical environment. Ecological Entomology, 39, 674-683.

Schleuning M, Blüthgen N, Flörchinger M, Braun J, Schaefer HM, Böhning-Gaese K (2011) Specialization and interaction strength in a tropical plant-frugivore network differ among forest strata. Ecology, 92, 26-36.
Shi L, Wang RW, Zhu LX, Zeng WM, Xu WL, Zheng Q (2011) Varying coefficient analysis for indeterminate species interactions with non-parametric estimation, exemplifying with a fig-fig wasp system. Chinese Science Bulletin, 56, 2545-2552.

Stouffer DB, Bascompte J (2010) Understanding food-web persistence from local to global scales. Ecology Letters, 13, 154-161.

Wang H, Ridley J, Dunn DW, Wang RW, Cook JM, Yu DW (2013) Biased oviposition and biased survival together help resolve a fig-wasp conflict. Oikos, 122, 533-540.

Wang ZJ (2010) Impact of Non-pollinating Fig Wasps on the Fig-fig Wasps Mutualism in Three Monoecious Ficus. PhD dissertation, University of the Chinese Academy of Sciences, Beijing. (in Chinese with English abstract) [王振吉 (2010) 非传粉榕小蜂对三种雌雄同株榕树一榕蜂互利共 生系统的影响. 博士学位论文, 中国科学院大学, 北京.]

Wang ZJ, Zhang FP, Peng YQ, Yang DR (2009) Reproductive strategy and impact on the fig-pollinator mutualism of one non-pollinating fig wasp species. Biodiversity Science, 17, 168-173. (in Chinese with English abstract) [王振吉, 张凤 萍, 彭艳琼, 杨大荣 (2009) 一种非传粉榕小蜂的繁殖策 略及其对榕-蜂互利共生系统的影响. 生物多样性, 17, 168-173.]

Wang ZM, Hu HY, Niu LM, Huang DW (2010) Population dynamics of 16 fig wasp species in Ficus benjamina. Acta Ecologica Sinica, 30, 3858-3864. (in Chinese with English abstract) [王仲敏, 胡好远, 牛黎明, 黄大卫 (2010) 垂叶 榕上16种榕小蜂的种群动态. 生态学报, 30, 3858-3864.]

Wasserman S, Faust K (1994) Social Network Analysis: Methods and Applications. Cambridge University Press, Cambridge.

Weiblen GD (2002) How to be a fig wasp. Annual Review of Entomology, 47, 299-330.

Williams RJ, Martinez ND (2000) Simple rules yield complex food webs. Nature, 404, 180-183.

Woodward G, Brown LE, Edwards FK, Hudson LN, Milner AM, Reuman DC, Ledger ME (2012) Climate change impacts in multispecies systems: Drought alters food web size structure in a field experiment. Philosophical Transactions of the Royal Society of London, 367, 2990-2997.

Zhang KY (1963) The climatic characteristics of southern Yunnan region and its formation mechanism. Acta Meteorologica Sinica, 33, 218-230. (in Chinese) [张克映 (1963) 滇南气候的特征及其形成因子的初步分析. 气象学报, 33, 218-230.]

(责任编委: 朱朝东 责任编辑: 时意专)

\section{附录 Supplementary Material}

\section{附录1 西双版纳地区垂叶榕榕小蜂的种类及其生物学特性}

Appendix 1 List of fig wasps and their morphological characteristics of Ficus benjamina in Xishuangbanna http://www.biodiversity-science.net/fileup/PDF/2019294-1.pdf 
董乙义, 彭艳琼, 王波. 垂叶榕榕小蜂群落及种间互作网络季节动态. 生物多样性, 2020, 28 (4): 496-503.

http://www.biodiversity-science.net/CN/10.17520/biods.2019294

附录1 西双版纳地区垂叶榕榕小蜂的种类及其生物学特性

Appendix 1 List of fig wasps and their morphological characteristics of Ficus benjamina in Xishuangbanna

\begin{tabular}{|c|c|c|}
\hline $\begin{array}{l}\text { 科/亚科 } \\
\text { Family/sub-family }\end{array}$ & $\begin{array}{l}\text { 物种 } \\
\text { Species }\end{array}$ & $\begin{array}{l}\text { 识别特征 } \\
\text { Identification notes }\end{array}$ \\
\hline Agaoninae/Agaoninae & $\begin{array}{l}\text { Eupristina } \\
\text { koningsbergeri }\end{array}$ & $\begin{array}{l}\text { 雌蜂体黑, 产卵器较体长更短; 雄蜂无翅 Female, body black, ovipositor } \\
\text { length shorter than body length; male, no wings }\end{array}$ \\
\hline $\begin{array}{l}\text { Pteromalidae/ } \\
\text { Epichrysomallina }\end{array}$ & Sycobia sp. 1 & $\begin{array}{l}\text { 雌蜂体棕色; 雄蜂头部纵脊隆突 Female, body brown; male, with longitudinal } \\
\text { carina on head }\end{array}$ \\
\hline $\begin{array}{l}\text { Pteromalidae/ } \\
\text { Epichrysomallina }\end{array}$ & Sycobia sp. 2 & $\begin{array}{l}\text { 雌蜂体黄色, 腹部带条纹; 雄蜂有翅 Female, body yellow, stripes of the } \\
\text { gaster; male, with wings }\end{array}$ \\
\hline $\begin{array}{l}\text { Pteromalidae/ } \\
\text { Epichrysomallina }\end{array}$ & Sycobia sp. 3 & $\begin{array}{l}\text { 雌蜂深灰色, 头部黄色; 雄蜂有翅 Female, body dark grey and head yellow; } \\
\text { male, with wings }\end{array}$ \\
\hline $\begin{array}{l}\text { Pteromalidae/ } \\
\text { Epichrysomallina }\end{array}$ & Acophila sp. 1 & $\begin{array}{l}\text { 雌蜂体呈黑色, 产卵器较体长更短; 雄蜂触角黄色 Female, body black, } \\
\text { ovipositor length shorter than body length; male, antenna yellow }\end{array}$ \\
\hline $\begin{array}{l}\text { Pteromalidae/ } \\
\text { Sycoryctinae }\end{array}$ & $\begin{array}{l}\text { Philotrypesis } \\
\text { tridentate }\end{array}$ & $\begin{array}{l}\text { 雌蜂黄色, 体型较大, 产卵器短; 雄蜂无翅 Female, body yellow, stout and } \\
\text { robust, ovipositor length shorter than body length; male, no wings }\end{array}$ \\
\hline $\begin{array}{l}\text { Pteromalidae/ } \\
\text { Sycoryctinae }\end{array}$ & Philotrypesis sp. 1 & $\begin{array}{l}\text { 雌蜂体黑, 产卵器为体长 } 2 \text { 倍多 Female, body black, ovipositor length more } \\
\text { than } 2 \text { times of body length }\end{array}$ \\
\hline $\begin{array}{l}\text { Pteromalidae/ } \\
\text { Sycoryctinae }\end{array}$ & Philotrypesis sp. 2 & $\begin{array}{l}\text { 雌蜂体黄色, 产卵鞘前端膨大处与体长等长 Female, body yellow, the length } \\
\text { of anteriorly bulging of ovipositor sheath equal to body length }\end{array}$ \\
\hline $\begin{array}{l}\text { Pteromalidae/ } \\
\text { Sycoryctinae }\end{array}$ & Philotrypesis sp. 3 & 雌蜂黑色; 雄蜂略带黑色 Female, body black; male, body blackish \\
\hline $\begin{array}{l}\text { Pteromalidae/ } \\
\text { Sycoryctinae }\end{array}$ & Sycoscapter sp. 1 & $\begin{array}{l}\text { 雌蜂体黑绿色, 产卵器较体长更长; 雄蜂无翅 Female, body black green, } \\
\text { ovipositor length longer than body length; male, no wings }\end{array}$ \\
\hline $\begin{array}{l}\text { Pteromalidae/ } \\
\text { Otitesellinae }\end{array}$ & $\begin{array}{l}\text { Walkerella } \\
\text { benjamini }\end{array}$ & $\begin{array}{l}\text { 雌蜂体呈铜绿色, 产卵器短; 雄蜂无翅 Female, body metallic green, } \\
\text { ovipositor length shorter than body length; male, no wings }\end{array}$ \\
\hline $\begin{array}{l}\text { Pteromalidae/ } \\
\text { Otitesellinae }\end{array}$ & Walkerella sp. 1 & $\begin{array}{l}\text { 雌蜂体黑带铜蓝色, 产卵器向下; 雄蜂无翅 Female, body black and metallic } \\
\text { blue, ovipositor posture prolapse; male, no wings }\end{array}$ \\
\hline $\begin{array}{l}\text { Pteromalidae/ } \\
\text { Otitesellinae }\end{array}$ & Walkerella sp. 2 & $\begin{array}{l}\text { 雌蜂体淡黑色, 产卵器较体长更短; 雄蜂无翅 Female, body blackish, } \\
\text { ovipositor length shorter than body length; male, no wings }\end{array}$ \\
\hline Eurytomidae & Sycophila sp. 1 & $\begin{array}{l}\text { 雌蜂体黄色, 带黑色翅斑; 雄蜂有翅 Female, body yellow, black spots on the } \\
\text { wings; male, with wings }\end{array}$ \\
\hline Ormyridae & Ormyrus sp. 1 & $\begin{array}{l}\text { 雌蜂体铜绿色, 产卵器较体长更短; 雄蜂有翅 Female, body metallic green, } \\
\text { ovipositor length shorter than body length; male, with wings }\end{array}$ \\
\hline
\end{tabular}

